\title{
Focused Extracorporeal Shockwave Therapy in Physical Medicine and Rehabilitation
}

\author{
Richard Crevenna $^{1} \cdot$ Michael Mickel $^{1}$ • Othmar Schuhfried ${ }^{1}$ • Christina Gesslbauer ${ }^{1}$. Andrej Zdravkovic ${ }^{1}$. \\ Mohammad Keilani ${ }^{1}$
}

Accepted: 4 December 2020 / Published online: 18 December 2020

(C) The Author(s) 2020

\begin{abstract}
Purpose of Review Focused extracorporeal shockwave therapy (fESWT) is a physical treatment modality developed over the last 25 years for musculoskeletal indications. It has many indications in the field of physical medicine and rehabilitation (PM\&R) and is effective, time-efficient, and cost-efficient. This review focuses on basics and on clinical indications as well as on significant trends in fESWT.

Recent Findings In PM\&R, stimulation of healing processes in tendons, surrounding tissue, and bones via mechanotransduction seems to be a relevant biological effect. The International Society for Medical Shockwave Treatment (ISMST) describes different types of indications (such as approved standard indications - in accordance with most scientific evidence - like calcifying tendinopathy of the shoulder, plantar fasciitis lateral epicondylopathy of the elbow, greater trochanter pain syndrome, patellar tendinopathy, Achilles tendinopathy and bone non-union, common empirically tested clinical uses, exceptional or expert indications, and experimental indications).

Summary fESWT is a relevant treatment option in PM\&R and regenerative medicine. In recent years, historical paradigms (for example, application in cancer patients) have changed and new indications (such as nerve regeneration, myofascial trapezius syndrome, low back pain, dermatosclerosis, and lymphedema) are supported. Future translational research should focus on establishing actual exceptional indications and experimental indications for clinical routine.
\end{abstract}

Keywords Focused extracorporeal shockwave therapy $\cdot$ Mechanotransduction $\cdot$ Regeneration $\cdot$ Physical medicine and rehabilitation

\section{Introduction}

In the field of physical medicine and rehabilitation (PM\&R), several physical modalities are used for the prevention and treatment of different diseases and also for rehabilitation [1, 2]. Normally, these modalities are related to physical forces and energies such as mechanical forces (mechanotherapy), heat and cold (thermotherapy), electricity (electrotherapy), light (phototherapy), and baths/climate (balneology and climate therapy) and are able to induce physical and biophysiological reactions in the human body $[1,2]$. In most cases, they are applied

Richard Crevenna

richard.crevenna@meduniwien.ac.at

1 Department of Physical Medicine, Rehabilitation and Occupational Medicine, Medical University of Vienna, Waehringer Guertel 18-20, 1090 Vienna, Austria repeatedly and then are able to induce adaptive and regenerative modifications of physical functions $[1,2]$.

The focused extracorporeal shockwave therapy (fESWT) is a physical treatment modality from systematic field of mechanotherapy, namely the application of mechanical forces, and - at first implemented in medicine in the field of urology for the destruction of kidney stones - has been developed over the last 25 years also for musculoskeletal indications $[3-6,7 \cdot]$. In the last few years, it has been found that most effects of fESWT are related to mechanotransduction, with the idea that physical forces are able to influence tissue physiology and diseases, and lead to tissue regeneration and healing by pain relief, regulation of inflammation (immunomodulation), and induction of neoangiogenesis and of stem cell activities (migration, homing, differentiation) $[3-6,7 \cdot]$.

Therefore, fESWT can be seen as a modern, non-invasive treatment by application of shockwaves to injured tissue with 
the intention to reduce pain and to promote regeneration and healing processes. fESWT has been shown to be time-efficient and cost-efficient and, up today, is allowed to be applied only by qualified physicians. fESWT has many interesting indications, which are also located in the interdisciplinary field of PM\&R.

This narrative, short literature review focuses on basics and on clinical indications as well as on significant trends in fESWT.

\section{Basics and Effects of fESWT}

fESWT is related to repeatedly applied mechanical energies (shockwaves) and able to induce therapeutic regenerative modifications in the body.

Physically, a shockwave is an abrupt change in pressure with a velocity higher than the speed of sound in the medium where it propagates $[3-6,7 \cdot, 8,9]$. Thus, shockwaves are pressure waves with a very short rise time, very high pressure, followed by a wave of negative pressure, longer in duration but much lower in amplitude than the initial peak $[3-6,7 \cdot, 8,9]$.

fESWT is applied into small focal areas of $2-8 \mathrm{~mm}$ diameter in order to optimize therapeutic effects and reduce negative effects on the surrounding tissue. The physical effects seem to be dependent on the energy delivered to a certain area. The shockwave energy per unit area is called the energy flux density (in $\mathrm{mJ} / \mathrm{mm}^{2}$ ). It reflects the flow of energy in a perpendicular direction to the direction of dissemination. The energy flux density is an essential parameter of dosage of fESWT $[3-6,7 \cdot, 8,9]$.

Therapeutic focused shockwaves (used in fESWT) can be applied with low (energy flux density $<0.1 \mathrm{~mJ} / \mathrm{mm}^{2}$, as used in typical PM\&R indications) and high (energy flux density > $0.12 \mathrm{~mJ} / \mathrm{mm}^{2}$, as used in non-healing fractures and pseudoarthroses) energy levels.

The so-called "radial shockwaves" physically are not shockwaves but sound waves characterized by a diverging pressure field, which reaches maximal pressure at the source. This short review focuses only on fESWT, which show a pressure field that converges at a selected depth in the body tissues where the maximal pressure is reached. For fESWT, there are existing defined parameters such as energy density, maximum pressure, minimum pressure, and spatial expansion of the focus $[3-6,7 \cdot, 8,9]$. The different fESWT generator technologies used today use the electrohydraulic, electromagnetic, or piezoelectric effect principle. They are called electrohydraulic shockwave transducer, electromagnetic shockwave transducer, and piezoelectric shockwave transducer and can produce shockwaves at least at the focal point $[3-6,7 \cdot, 8,9]$.

The electrohydraulic principle is that in which the shockwaves are generated by a spark plug. The shockwaves spread in a medium (water) and are then focused in one place by a parabolic mirror $[3-6,7 \cdot, 8,9]$.

The piezoelectric principle is based on a high-voltage discharge across a pattern of piezoelectric crystals. Each element expands, generating a pressure pulse that spreads toward the focal region, of the arrangement. The placement of the crystals and the shape of the sphere focus the shockwave into the tissue $[3-6,7 \cdot, 8,9]$.

Electromagnetic shockwaves are generated by an electric current that passes through a coil. This produces a magnetic field, which produces a shockwave via the conductive membrane in surrounding water. Acoustic lenses are used to focus the shockwave into the tissue $[3-6,7 \cdot, 8,9]$.

This fESWT is allowed to be applied only by qualified physicians. The so-called radial "shockwave" (sound wave) generators produce only sound waves and are allowed to be used also by qualified therapists [4].

fESWT was primarily used in the field of urology to disintegrate urolithiasis (so-called lithotripsy). In musculoskeletal medicine (PM\&R, traumatology and orthopedics), fESWT aims to induce adaptation, tissue repair, and regeneration $[3-6,7 \cdot, 8,9]$. Thus, fESWT is a kind of adaptive and regenerative medicine $[3-6,7 \cdot, 8,9]$. As there are several approved standard indications and common empirically tested clinical uses now, the exact mechanisms of fESWT (reversal of chronic inflammation, dissolution of calcified fibroblasts, stimulation of collagen production, dispersion of substance $\mathrm{P}$ as a pain mediator, and release of trigger points) are still under exploration $[3-6,7 \cdot, 8,9]$.

Nevertheless, the term of mechanotransduction seems to be important. The biophysical cellular mechanotransduction through cytoskeleton into nuclei leads to regulation of gene expression and causes relevant biological effects, which lead to stimulation of healing processes in tendons, surrounding tissue, and bones [3-6, 7•, 8, 9].

Knowledge about mechanism is growing $[3-6,7 \cdot, 8,9]$. For example, d'Agostino et al. [3] described in a comprehensive review in more detail that fESWT has been described in experimental studies to reduce expression of several metalloproteinases (enzymes capable of degrading the collagen) and inflammatory interleukins and, in turn, to upregulate gene expression of typical tendon markers (such as scleraxis) and anti-inflammatory cytokines (e.g., interleukin-10).

In animal experiments, fESWT was shown to promote healing of "collagenase - Achilles tendinitis," by inducing transforming growth factor beta 1 and insulin-like growth factor I. Histological observations demonstrated that fESWT resolved swelling and edema as well as inflammatory cell infiltration in affected tendons [3].

Furthermore, at the bone level, after fESWT exposure, there has been described early expression of angiogenesisrelated growth factors, including endothelial nitric oxide synthase, vascular endothelial growth factor, and proliferating cell 
nuclear antigen, therefore producing new vessel in-growth with improved blood supply as well as increasing cell proliferation [3].

Furthermore, shockwaves have been shown to be able to enhance regeneration of damaged tissue by leading to migration, homing, and differentiation of human stem cells $[3-6,7 \cdot$, $8,9]$.

fESWT is commonly used in the management of musculoskeletal disorders, and there is increasing evidence for its clinical effectiveness. Pain relief, destruction of calcifications, and the described mechanotransduction-initiated tissue regeneration and further remodeling processes seem to be the most important working mechanisms [3-6, 7•, 8, 9]. Clinical pain reduction is often followed by changes in imaging [10]. In this regard, Al-Abbad et al. [10] performed recently a metaanalysis with meta-regression on imaging outcomes for rotator cuff calcific tendinitis, osteonecrosis of the femoral head, and plantar fasciitis. There was an overall reduction in the size of measured lesion following fESWT for calcium deposit diameter, lesion size in femoral head osteonecrosis, and plantar fascia thickness [10].

The practical procedure of fESWT in clinical routine is easy-the International Society for Medical Shockwave Treatment (ISMST) clearly recommends standard examinations including history taking and clinical examination, radiological imaging, and neurological and/or laboratory diagnostic tests before fESWT, and that only qualified physicians should apply fESWT $[5,7 \bullet]$.

If there is an indication for fESWT and before application of fESWT, the physician has to explain the method of fESWT and of other possible treatment options. For example, other possible non-operative treatment options for calcifying tendinitis of the shoulder could be further physical modalities and corticosteroid injection. Clinical target of therapy is to relieve pain as well as improvement of function of upper extremity. When non-operative treatment over a prolonged period fails, surgical treatment should be considered $[5,7 \bullet]$.

If the patient decides to get fESWT, written informed consent has to be obtained by the patient. After this fESWT can be started, it is important to remember that fESWT has to be applied by a physician ("...is carried out by the doctor!...") $[5,7 \bullet]$. The shockwaves are applied via a contact gel through the skin, and even if fESWT is painful, no local or regional anesthesia is necessary. The adjustment is made before and during the treatment by means of a clinical examination, pain description (of the patient), and/or imaging. A fixed position is important; thus, a possible interruption of fESWT (pain) would be possible any time. During one fESWT session, 1000-3000 shockwaves are applied with a frequency of 1-5 impulses per second. The duration of one session is about 10 min up to 60 min including history taking and clinical diagnostics, and one or more treatments can be necessary depending on indication $[5,7 \cdot]$. Exact choice of the parameters time, frequency, duration, and intensity of fESWT depend on the underlying disease and, in turn, on the patient's pain tolerance during treatment sessions $[5,7 \bullet]$.

During treatment, all data are recorded and documented precisely. After treatment, medication (e.g., analgesics) or cooling may be recommended for the post-treatment period $[5,7 \bullet]$.

For example and due to the fact that this was the first approved indication by the U.S. Food and Drug Administration (FDA), fESWT for treatment of plantar fasciitis (synonyms include plantar heel spur, fasciitis plantaris, plantar heel pain) is described according to the ISMST procedure [7•]. fESWT is described to be provided personally by a doctor qualified by means of specialist knowledge [7•]. In plantar fasciitis, fESWT is applied by using a focused shockwave source with applicator with an intensity of $0.08-0.35 \mathrm{~mJ} / \mathrm{mm}^{2}$ (pain-adapted dosingfeedback!) and by using ultrasound gel as coupling medium via skin at the relevant (patient-oriented focusing) location (feedback!). No local anesthesia is needed, but sometimes if necessary, cryotherapy [7•]. The fESWT is applied in a frequency up to $5 \mathrm{~Hz}$ with $1500-2000$ impulses per session. Up to 5 treatments with an interval of 1-2 weeks are performed [7•].

After fESWT, a documentation of treatment parameters and a monitoring of clinical and circulatory function have to be performed. Possible complications (adverse or side effects) such as hematoma, pain enhancement, and nerve irritation have to be documented and treated. For the post-treatment period, analgesics or cooling can be recommended [7•]. For follow-up treatment, counseling concerning stretching exercises and individual physiotherapy and sports adaptation are important [7•]. The first clinical success control makes sense 4-6 weeks after the fESWT, but with respect to the clinical relevant phenomena of mechanotransduction after 12 weeks (3 months) $[5,7 \bullet]$.

\section{Side Effects of fESWT}

If the application of fESWT is done in a proper way-namely performed by a qualified physician after thorough history taking, clinical examination, radiological imaging, and neurological and/or laboratory-diagnostic tests/investigations (if necessary), the typical side effects from extracorporeal shockwave therapy (ESWT) are limited to skin reddening, mild bruising, swelling, pain, numbness, or tingling in (or around) the treated area during and up to $24 \mathrm{~h}$ after treatment $[4-6,7 \bullet]$. Other described side effects seem to be very rare $[4-6,7 \cdot, 11]$. Nevertheless, fESWT should not be applied after extensive pretreatment with corticoids due to the fact that this can lead to ruptures of tendons $[4-6,7 \bullet]$. 


\section{Contraindications of fESWT}

The contraindications differ in fESWT with low energy and high energy with additional contraindications [5, 7•].

In fESWT with low energy, there are existing contraindications if the fetus (in pregnant women) or a malignant tumor (but not cancer as an underlying disease) is in the treatment area $[5,7 \cdot]$.

Beside this (fetus or a malignant tumor in the treatment area), fESWT with high-energy focused waves has additional contraindications such as lung tissue in the treatment area, epiphyseal plate in the treatment area, brain or spine in the treatment area, and severe coagulopathy and "marcu marization" $[5,7 \bullet]$. Furthermore, fESWT application during acute infection should be avoided.

\section{Myths and Paradigms in fESWT}

Some paradigms have changed in the last decades $[5,12 \cdot$, 13•]. Lohrer et al. [12•] were able to revise different historical paradigms as set in a historical consensus meeting on ESWT in 1995. Examples are the differences between fESWT with radial sound waves, and also parameters such as energy intensity, focus size/lesion size, regional/local anesthesia, imagingguided focusing, the application near/on growth plates, stage and chronicity/acuteness, calcifications, and number of sessions [12•]. The size of the lesion (tissue area to be treated with fESWT) can be small or large. Direct clinical feedback from the patient seems to be superior to imaging-guided focusing. fESWT can be applied without the risk of epiphyseal damage in juvenile patients (apophyseal osteochondral lesions in patients with open growth plates). The authors mention that fESWT protocols should be adapted to the stage and chronicity of the treated lesion, and encourage further research to study the management of acute injuries [12•]. It seems that different stages of a given pathology/disease will respond differently to fESWT. Modern musculoskeletal fESWT is performed with energy below $0.28 \mathrm{~mJ} / \mathrm{mm}^{2}$ and without anesthesia $[4-6,7 \bullet, 12 \bullet]$. Nevertheless, several treatment parameters remain still relying on empirical data, and more translational research is urgently needed $[4-6,7 \bullet, 12 \bullet]$.

In former times, radial sound waves as well as focused shockwaves have been applied only by interested physicians. Today, qualified therapists are allowed to apply radial sound waves.

Thus, the application of real shockwaves, namely fESWT, remains the domain of qualified physicians $[4-6,7 \cdot, 13 \bullet]$.

\section{Historical contraindications}

The application of fESWT has been regarded as a contraindication for patients suffering from cancer until $2016[5,13 \bullet]$. In
October 2016, the ISMST updated clinical recommendations for the use of therapeutic shockwaves in clinical practice based on an assessment of the currently published scientific and clinical information and accepted approaches to treatment, namely to clarify indications and contraindications for the use of fESWT $[5,13 \bullet]$. For patients suffering from cancer, the situation has changed since this update. Cancer itself-in the form of an underlying malignant disease - is not a contraindication for ESWT, but malignant tumors, metastasis, multiple myeloma, and lymphoma in the treatment area, and active leukemia and leukemic phase of lymphoma (not in remission) have to be seen as contraindications for treatment with fESWT with low and high energy levels [5, 13•].

\section{Indications of fESWT}

As described, fESWT is a non-invasive treatment option by application of shockwaves to injured (soft) tissue with the intention to reduce pain and to promote healing processes in tendinopathies and bone and skin pathologies $[3-6,7 \cdot, 8,14$, 15]. The application of fESWT in musculoskeletal disorders started with over-use tendinopathies (plantar fasciitis, lateral epicondylitis, calcific or non-calcific tendonitis of the shoulder) with an excellent success rate (ranged from 65 to $91 \%$ ) and negligible side effects and complications $[3-6,7 \cdot, 8,14$, 15]. In the year 2000, FDA (USA) approved fESWT for the treatment of proximal plantar fasciitis as first indication [3-6, $7 \cdot, 8,14,15]$. Furthermore, fESWT was applied in non-union of long bone fracture, avascular necrosis of femoral head, chronic diabetic (and non-diabetic) wounds, and ulcers [3-6, $7 \cdot, 8,14,15]$.

The ISMST defined approved standard indications for fESWT [4-6, 7•]. They include tendinopathies; bone and skin pathologies, such as plantar fasciitis, with or without heel spur; calcifying tendinopathy of the shoulder; bone non-union (non-healing fractures) or pseudoarthroses; non-healing wounds; lateral epicondylopathy of the elbow or tennis elbow; Achilles tendinopathy; and more (Table 1) $[4-6,7 \cdot]$.

Furthermore, there are existing so-called common empirically tested clinical uses such as different other tendinopathies, myofascial syndrome, and bone marrow edema (Table 1) $[4-6,7 \bullet]$.

Almost all of the indications of these both groups are part of the daily clinical routine of physiatrist and/or of interdisciplinary treatment or rehabilitation concepts guided by physiatrist (Table 1) [4-6, 7•, 16, 17•, 18-49].

The guidelines of the ISMST (published in October 2016) provide scientific literature according to evidence-based criteria.

In regard to the current evidence of fESWT for the approved indications, a comprehensive review by Moya et al. 
Table 1 Approved standard indications and common empirically tested clinical uses as described by the ISMST $[4-6,7 \cdot, 16-49]$

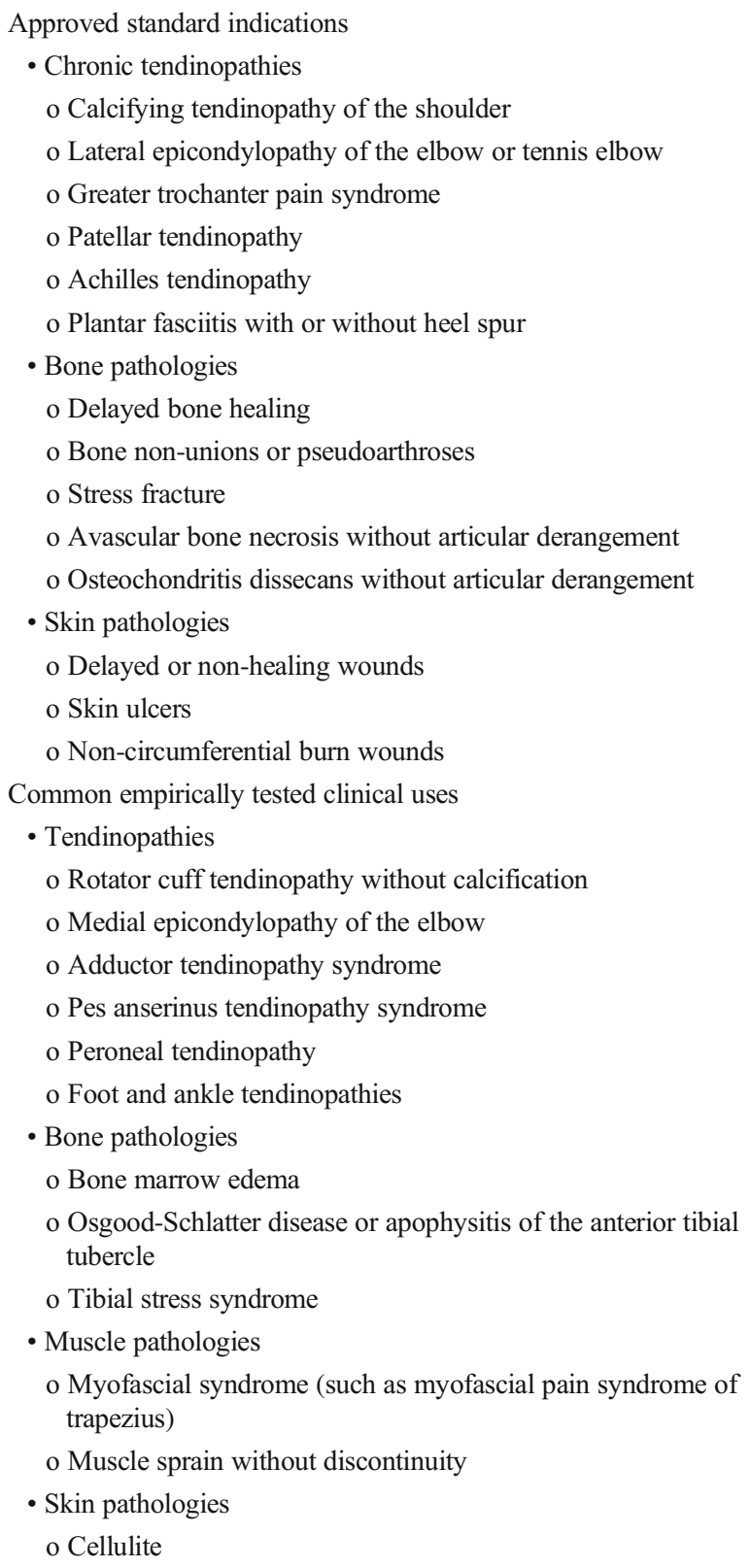

[17.] on the role of ESWT in musculoskeletal disorders reported good evidence for the use of ESWT in calcifying tendinopathy of the shoulder and plantar fasciitis (grade A recommendation $=$ level I studies with consistent findings). Grade B recommendation (level II or III studies with consistent findings) was found for lateral epicondylopathy of the elbow, greater trochanter pain syndrome, patellar tendinopathy, Achilles tendinopathy, and bone non-union [17•].

In summary, there was effectiveness in pain reduction and functional outcomes and in the resorption rate of calcification
[17•]. The best evidence for the usage of fESWT was found with low energy levels for tendon disorders and with a high energy level for calcifying tendinopathy as well as bone pathologies [17•]. However, because of the variability in the treatment protocols, further high-quality studies are required to standardize the optimal fESWT approach for clinical decision-making [17•].

A systematic review of systematic reviews on clinical management of tendinopathy regardless of tendinopathy type or location was published in 2020. Hereby, fESWT demonstrated moderate effectiveness to improve pain and function [19].

In a further recently published systematic review on ESWT in upper limb diseases including rotator cuff tendinopathy, subacromial impingement, and medial and lateral epicondylitis, 26 studies were included. In 24 studies, fESWT was used [21]. The authors concluded that ESWT is a safe and effective treatment for all indications investigated [21]. However, there does not seem to be a consensus about energy (low vs. high energy) levels and the number of sessions of ESWT required, as well [21]. In the studies included, the protocols provided 1-6 sessions (with an interval of 12 weeks); however, increasing the number of therapy sessions seems not to improve the outcome [21].

Concerning avascular bone necrosis, a meta-analysis of effect of fESWT concluded that fESWT might be a safe and effective means to relieve pain and improve motor function in lower limb [44].

A recent meta-analysis investigating fESWT in patients suffering from diabetic foot ulcers showed that fESWT significantly improved wound healing by increasing wound epithelialization and reducing wound surface area, as well [39].

Recent meta-analysis on common empirically tested clinical uses is evident, for example myofascial pain syndrome of trapezius [47, 48]. Hereby, there was a significant improvement of neck pain for the short term. Nevertheless, the limited quality of these studies indicates the need for further studies in this area.

The so-called exceptional indications or expert indications are only for experienced users and include, for example, osteoarthritis, trigger finger, erectile dysfunction, polyneuropathy, and lymphedema (Table 2) [4-6, 7•].

Examples for experimental indications are heart muscle ischemia, osteoporosis, and complex regional pain syndrome (CRPS). There is an urgent need for research to increase knowledge and scientific evidence about the usability of fESWT in these indications (Table 2) [4-6, 7•]. Thus, to establish exceptional indications and experimental indications for regular clinical routine, more translational research and clinical studies are urgently needed $[4-6,7 \cdot, 50-70]$. The field of PM\&R seems to be an ideal place for this research. 
Table 2 Exceptional or expert indications and experimental indications as described by the ISMST $[4-6,7 \cdot, 50-70]$

\author{
Exceptional indications-expert indications \\ - Musculoskeletal pathologies \\ o Osteoarthritis \\ o Dupuytren's disease \\ o Plantar fibromatosis or Ledderhose disease \\ o De Quervain's disease \\ o Trigger finger \\ - Neurological pathologies \\ o Spasticity \\ o Polyneuropathy \\ o Carpal tunnel syndrome \\ - Urologic pathologies \\ o Pelvic chronic pain syndrome (abacterial prostatitis) \\ o Erectile dysfunction \\ o Peyronie's disease \\ - Others \\ o Lymphedema \\ Experimental indications \\ - Heart muscle ischemia \\ - Peripheral nerve lesions \\ - Pathologies of the spinal cord and brain \\ - Skin calcinosis \\ - Periodontal disease \\ - Jawbone pathologies \\ - Complex regional pain syndrome \\ - Osteoporosis
}

\section{Significant Trends and Aspects of fESWT in the Field of PM\&R}

Tendinopathies, calcified tissue, carpal tunnel syndrome, polyneuropathy, and delayed or non-healing wounds such as skin ulcers but also lymphedema and dermatosclerosis are typical indications which are treated by using different combinations of modalities of the field of PM\&R. fESWT has been shown to be a very effective option of mechanotherapy to accelerate regeneration and healing and thus to improve the functional status of patients. fESWT is easy and safe to apply and, therefore, should be part of the therapeutic portfolio of every physiatrist $[1,13 \bullet]$.

In the planning of rehabilitation (for almost all indications) concepts, fESWT can be an option for necessary initial pain reduction with the aim to better mobilize patients and to enable them to easily attend active physiotherapy and systematic exercise programs [13•]. Since 2016, fESWT is allowed to be applied to cancer survivors and to be part of cancer rehabilitation which has been shown to be an effective option to accelerate return to work and improve the work ability and social participation of cancer survivors $[13 \bullet, 71,72]$. Furthermore, regular active exercise-as part of cancer rehabilitation - with the intention to improve muscular strength, endurance capacity, sensorimotor functions, and flexibility has been shown to increase cancer-specific survival $[13 \cdot, 71,72]$. Before starting exercise programs, musculoskeletal conditions, such as plantar fasciitis, calcific tendinitis of the shoulder, tennis elbow, and other musculoskeletal pain syndromes, have to be treated by using effective methods to reduce bodily pain and to mobilize and enable the patient to participate actively [13•] (Fig. 1). fESWT has been shown to be an effective method for treatment of these indications [4-6, 7•, 13•]. Until 2016, the application of fESWT has been seen as a contraindication for cancer patients (cancer survivors). After the described change in paradigm, fESWT is only contraindicated at the tumor site $[5,13 \bullet]$. Due to the fact that some physicians still see cancer as a general contraindication for the use of fESWT, many cancer patients remain undertreated and therefore cannot be mobilized due to bodily pain [13•].

In cancer survivors, there are typical cancer- or treatmentrelated side effects to overcome during cancer rehabilitation such as polyneuropathy, lymphedema, musculoskeletal pain, reduced physical performance, and erectile dysfunction [72]. As a part of multimodal rehabilitation concepts, fESWT can be used as an effective and, in most cases, time-efficient option against these side effects which have been recommended by the ISMST as exceptional indications or expert indications $[5,13 \bullet]$. Nevertheless, further research is needed to identify the relevant parameters such as the number of sessions, energy transmitted, and frequency to optimize the application fESWT for specific indications in cancer rehabilitation.

The treatment of lymphedema in cancer patients is, at the moment, an expert indication (a so-called off-label indication) with mechanotransduction and stimulation of collagen production to be the therapeutic principles with the aim to stop dermatosclerosis and to increase lymphangiogenesis. A

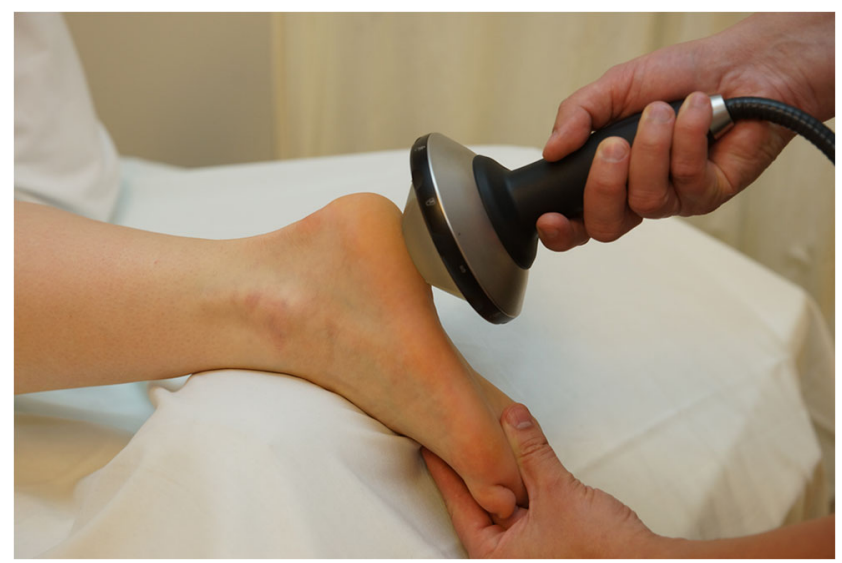

Fig. 1 fESWT in a cancer patient suffering from plantar fasciitis with the intention of mobilization to enable to regular exercise [13•] 
superficial area-wide fESWT treatment of the affected limbs was suggested [50, 51].

fESWT for treatment of polyneuropathy in cancer patients is, at the moment, an expert indication, a so-called off-label use where mechanotransduction seems to be the therapeutic principle. The treatment goals are pain reduction, neuroregeneration, and angiogenesis. A superficial area-wide fESWT treatment of the affected limbs was suggested $[6,7 \cdot$, 52]. To our opinion, also the (effective) fESWT of erectile dysfunction should also be part of a holistic cancer rehabilitation concept after radical prostatectomy but remains a domain of the urologist within an interdisciplinary cancer rehabilitation concept [13•, 54-63].

Sexual function is a part of human health. In penile rehabilitation, fESWT has been shown to be able to improve erectile function in patients with vascular phosphodiesterase type 5 inhibitor (PDE5I) refractory erectile dysfunction $[6,7 \bullet$, 54-63].

Park et al. [65] were able to show that fESWT is able to enhance peripheral nerve remyelination and gait function in a nerve crush model. Nevertheless, long-term follow-up and studies about the molecular mechanisms will be needed to confirm these effects [65].

For fESWT, there are many very interesting and promising indications from the field of PM\&R such as osteoarthritis, osteoporosis, nerve regeneration (carpal tunnel syndrome, polyneuropathy, spasticity after stroke), myofascial trapezius syndrome, low back pain, dermatosclerosis, chronic ulcers, and lymphedema which have to be established with high scientific evidence for clinical routine [50-53, 66-70]. Therefore, it seems that the field of PM\&R could be one of the best places for further research concerning fESWT, namely translational and clinical research to establish actual exceptional (expert) indications and experimental indications for regular clinical routine. Furthermore, the economic advantages of this treatment method should also be studied.

\section{Personal Observations and Views Concerning fESWT}

At the Department of Physical Medicine, Rehabilitation and Occupational Medicine, Medical University of Vienna, Austria, we are using ESWT (sound waves and shockwaves/ fESWT) since 2000 and have experiences in the treatment of different musculoskeletal indications such as plantar heel, calcified shoulder, tennis elbow, piriformis syndrome, and bone marrow edema, but also dermatological (ulcers) as well as neurological indications such as carpal tunnel syndrome $[13 \cdot, 73,75,76]$. Also in our experience, fESWT has been shown to be easy to apply, safe, clinically effective, and timeand cost-efficient in most cases. Furthermore, we were able to show that fESWT can be easily integrated into our outpatient treatment plans and rehabilitation concepts $[13 \cdot, 72,73,76]$.

Furthermore, (sound waves and) shockwaves seem to be very promising to treat indications from dermatology such as ulcers, lymphedema, dermatosclerosis, and a special dermatoimmunologic indication (for example, granuloma annulare) in our experience $[74,75]$.

The application of fESWT in special patient groups such as cancer patients and patients suffering from hemophilia is also part of the clinical routine in our specialized outpatient clinics for cancer rehabilitation and for hemophilia patients. Cancer patients have been shown to benefit from fESWT in musculoskeletal indications (plantar fasciitis, calcified shoulder) with the intention of mobilization to enable them to exercise for tertiary preventive purposes during cancer rehabilitation. As described, the treatment of lymphedema and polyneuropathy are very interesting expert indications in this patient group $[6,7 \bullet, 13 \bullet, 72]$. We were able to show that fESWT application can be safe in patients suffering from hemophilia but it should only be treated by physicians with a specialized expertise in this patient group [76].

At the moment, our clinical research group focuses on research of fESWT application in carpal tunnel syndrome, polyneuropathy, and lymphedema.

\section{Conclusions}

The fESWT has been developed over the last 25 years for musculoskeletal indications and is a physical non-invasive treatment modality from the part of mechanotherapy which has many indications in the field of PM\&R. Historical paradigms have changed (such as application in cancer patients and other contraindications), and new indications could be found.

In most cases, fESWT is repeatedly applied and then able to reduce pain and to induce adaptive and regenerative modifications of physical functions. fESWT is allowed to be applied only by qualified physicians and is effective and timeand cost-efficient. Mechanotransduction, the stimulation of adaptive and healing processes in tendons, surrounding tissue, and bones via mechanotransduction, seems to be a relevant biological effect of this relevant treatment option in regenerative medicine.

In PM\&R, fESWT leads to symptom (pain) reduction in different musculoskeletal conditions and to increase flexibility and mobility of patients, allowing activities such as walking, running, and lifting as the basis of regular, systematic exercise. To our opinion, the fESWT should be part of the therapeutic portfolio of every physiatrist. Significant future trends of fESWT in the field of PM\&R are the integration in treatment and rehabilitation concepts, and the rollout into patient groups for whom fESWT has been a contraindication in former years. 
Furthermore, actual exceptional indications and experimental indications should be established for regular clinical routine. For this purpose, more translational research and clinical research are urgently needed.

Funding Open Access funding provided by Medical University of Vienna.

\section{Compliance with Ethical Standards}

Conflict of Interest The authors declare that they have no competing interests.

Human and Animal Rights and Informed Consent All reported studies/ experiments with human or animal subjects performed by the authors have been previously published and complied with all applicable ethical standards (including the Helsinki Declaration and its amendments, institutional/national research committee standards, and international/national/institutional guidelines).

Open Access This article is licensed under a Creative Commons Attribution 4.0 International License, which permits use, sharing, adaptation, distribution and reproduction in any medium or format, as long as you give appropriate credit to the original author(s) and the source, provide a link to the Creative Commons licence, and indicate if changes were made. The images or other third party material in this article are included in the article's Creative Commons licence, unless indicated otherwise in a credit line to the material. If material is not included in the article's Creative Commons licence and your intended use is not permitted by statutory regulation or exceeds the permitted use, you will need to obtain permission directly from the copyright holder. To view a copy of this licence, visit http://creativecommons.org/licenses/by/4.0/.

\section{References}

Papers of particular interest, published recently, have been highlighted as:

- Of importance

1. https://www.aapmr.org/about-physiatry/about-physical-medicinerehabilitation (Accessed August 4, 2020).

2. Crevenna R. Physical medicine and rehabilitation-a relevant interdisciplinary speciality. Wien Med Wochenschr. 2016;166(1-2):23. https://doi.org/10.1007/s10354-015-0412-3.

3. d'Agostino MC, Craig K, Tibalt E, Respizzi S. Shock wave as biological therapeutic tool: from mechanical stimulation to recovery and healing, through mechanotransduction. Int J Surg. 2015;24(Pt B):147-53. https://doi.org/10.1016/j.ijsu.2015.11.030.

4. https://www.shockwavetherapy.org/about-eswt/physicalprinciples-of-eswt/ (Accessed August 04, 2020).

5. https://www.shockwavetherapy.org/fileadmin/user_upload/ dokumente/PDFs/Formulare/ISMST_consensus_statement_on indications_and_contraindications_20161012_final.pdf (Accessed August 04, 2020).

6. https://www.shockwavetherapy.org/about-eswt/indications/ (Accessed August 04, 2020).

7. https://www.shockwavetherapy.org/fileadmin/user_upload/ ISMST Guidelines.pdf (Accessed August 04, 2020). This guideline represents an overview on physics and technology as well as the practical application of extracorporeal shockwave therapy.

8. Notarnicola A, Moretti B. The biological effects of extracorporeal shock wave therapy (eswt) on tendon tissue. Muscles Ligaments Tendons J. 2012;2(1):33-7.

9. Liu DD, Ullah M, Concepcion W, Dahl JJ, Thakor AS. The role of ultrasound in enhancing mesenchymal stromal cell-based therapies. Stem Cells Transl Med. 2020;9(8):850-66. https://doi.org/10.1002/ sctm.19-0391.

10. Al-Abbad H, Allen S, Morris S, Reznik J, Biros E, Paulik B, et al. The effects of shockwave therapy on musculoskeletal conditions based on changes in imaging: a systematic review and metaanalysis with meta-regression. BMC Musculoskelet Disord. 2020;28(21(1)):275.

11. Liu HM, Chao CM, Hsieh JY, Jiang CC. Humeral head osteonecrosis after extracorporeal shock-wave treatment for rotator cuff tendinopathy. A case report. J Bone Joint Surg Am. 2006;88(6):1353-6. https://doi.org/10.2106/JBJS.E.00868.

12. Lohrer H, Nauck T, Korakakis V, Malliaropoulos M. Historical ESWT paradigms are overcome: a narrative review. Biomed Res Int. 2016;2016:3850461. https://doi.org/10.1155/2016/3850461 This paper describes the change of historical extracorporeal shockwave treatment paradigms.

13. Crevenna R, Mickel M, Keilani M. Extracorporeal shock wave therapy in the supportive care and rehabilitation of cancer patients. Support Care Cancer. 2019;27(11):4039-41. https://doi.org/10. 1007/s00520-019-05046-y This is an important review concerning focused extracorporeal shockwave therapy in cancer patients.

14. Wang CJ. Extracorporeal shockwave therapy in musculoskeletal disorders. J Orthop Surg Res. 2012;7:11. https://doi.org/10.1186/ 1749-799X-7-11.

15. Saggini R, Di Stefano A, Saggini A, Bellomo RG. Clinical application of shock wave therapy in musculoskeletal disorders: part I. J Biol Regul Homeost Agents. 2015;29(3):533-45.

16. Costa ML, Shepstone L, Donell ST, Thomas TL. Shock wave therapy for chronic Achilles tendon pain: a randomized placebocontrolled trial. Clin Orthop Relat Res. 2005;440:199-204. https://doi.org/10.1097/01.blo.0000180451.03425.48.

17. Moya D, Ramón S, Schaden W, Wang CJ, Guiloff L, Cheng JH. The Role of extracorporeal shockwave treatment in musculoskeletal disorders. J Bone Joint Surg Am. 2018;100(3):251-63 This is a review summarizing the current understanding of the treatment of musculoskeletal conditions with extracorporeal shochwave treatment.

18. Dedes V, Stergioulas A, Kipreos G, Dede AM, Mitseas A, Panoutsopoulos GI. Effectiveness and safety of shockwave therapy in tendinopathies. Mater Soc. 2018;30(2):131-46. https://doi.org/ 10.5455/msm.2018.30.141-146.

19. Irby A, Gutierrez J, Chamberlin C, Thomas SJ, Rosen AB. Clinical management of tendinopathy: a systematic review of systematic reviews evaluating the effectiveness of tendinopathy treatments. Scand J Med Sci Sports. 2020. https://doi.org/10.1111/sms.13734.

20. Reilly JM, Bluman E, Tenforde AS. Effect of shockwave treatment for management of upper and lower extremity musculoskeletal conditions: a narrative review. PM R. 2018;10(12):1385-403. https:// doi.org/10.1016/j.pmrj.2018.05.007.

21. Testa G, Vescio A, Perez S, Consoli A, Costarella L, Sessa G, et al. Extracorporeal shockwave therapy treatment in upper limb diseases: a systematic review. J Clin Med. 2020;9(2):53. https://doi. org $/ 10.3390 / \mathrm{jcm} 9020453$.

22. Wu KT, Chou WY, Wang CJ, Chen CY, Ko JY, Chen PC, et al. Efficacy of extracorporeal shockwave therapy on calcified and noncalcified shoulder tendinosis: a propensity score matched analysis. Biomed Res Int. 2019;2019:2958251-8. https://doi.org/10. $1155 / 2019 / 2958251$. 
23. Carlisi E, Lisi C, Dall'angelo A, Monteleone S, Nola V, Tinelli C, et al. Focused extracorporeal shock wave therapy combined with supervised eccentric training for supraspinatus calcific tendinopathy. Eur J Phys Rehabil Med. 2018;54(1):41-7. https:// doi.org/10.23736/S1973-9087.16.04299-4.

24. Gerdesmeyer L, Wagenpfeil S, Haake M, Maier M, Loew M, Wörtler K, et al. Extracorporeal shock wave therapy for the treatment of chronic calcifying tendonitis of the rotator cuff. A randomized controlled trial. JAMA. 2003;290(19):2573-80. https://doi. org/10.1001/jama.290.19.2573.

25. Korakakis V, Whiteley R, Tzavara A, Malliaropoulos N. The effectiveness of extracorporeal shockwave therapy in common lower limb conditions: a systematic review including quantification of patient-rated pain reduction. Br J Sports Med. 2018;52(6):387407. https://doi.org/10.1136/bjsports-2016-097347.

26. Mitchkash M, Robinson D, Tenforde AS. Efficacy of extracorporeal pulse-activated therapy in the management of lower-extremity running-related injuries: findings from a large case cohort. J Foot Ankle Surg. 2020;59(4):795-800. https://doi.org/10.1053/j.jfas. 2020.02.008

27. Ramon S, Russo S, Santoboni F, Lucenteforte G, Di Luise C, de Unzurrunzaga R, et al. Focused shockwave treatment for greater trochanteric pain syndrome: a multicenter, randomized, controlled clinical trial. J Bone Joint Surg Am. 2020;102(15):1305-11. https:// doi.org/10.2106/JBJS.20.00093.

28. Carlisi E, Cecini M, Di Natali G, Manzoni F, Tinelli C, Lisi C. Focused extracorporeal shock wave therapy for greater trochanteric pain syndrome with gluteal tendinopathy: a randomized controlled trial. Clin Rehabil. 2019;33(4):670-80. https://doi.org/10.1177/ 0269215518819255

29. Zhang ZJ, Lee WC, Fu SN. One session of extracorporeal shockwave therapy-induced modulation on tendon shear modulus is associated with reduction in pain. J Sports Sci Med. 2020;19(2):30916.

30. Everhart JS, Cole D, Sojka JH, Higgins JD, Magnussen RA, Schmitt LC, et al. Treatment options for patellar tendinopathy: a systematic review. Arthroscopy. 2017;33(4):861-72. https://doi. org/10.1016/j.arthro.2016.11.007.

31. Jarin I, Bäcker HC, Vosseller JT. Meta-analysis of noninsertional Achilles tendinopathy. Foot Ankle Int. 2020;41(6):744-54. https:// doi.org/10.1177/1071100720914605.

32. Stania M, Juras G, Chmielewska D, Polak A, Kucio C, Król P. Extracorporeal shock wave therapy for Achilles tendinopathy. Biomed Res Int. 2019;2019:3086910-3. https://doi.org/10.1155/ 2019/3086910

33. Wheeler PC, Tattersall C. Novel interventions for recalcitrant Achilles tendinopathy: benefits seen following high-volume image-guided injection or extracorporeal shockwave therapy-a prospective cohort study. Clin J Sport Med. 2020;30(1):14-9. https:// doi.org/10.1097/JSM.0000000000000580.

34. Hsu YC, Wu WT, Chang KV, Han DS, Chou LW. Healing of Achilles tendon partial tear following focused shockwave: a case report and literature review. J Pain Res. 2017;10:1201-6. https:// doi.org/10.2147/JPR.S132951.

35. Zhang S, Li H, Yao W, Hua Y, Li Y. Therapeutic response of extracorporeal shock wave therapy for insertional Achilles tendinopathy between sports-active and nonsports-active patients with 5-year follow-up. Orthop J Sports Med. 2020;8(1): 2325967119898118. https://doi.org/10.1177/2325967119898118.

36. Li H, Xiong Y, Zhou W, Liu Y, Liu J, Xue H, et al. Shock-wave therapy improved outcome with plantar fasciitis: a meta-analysis of randomized controlled trials. Arch Orthop Trauma Surg. 2019;139(12):1763-70. https://doi.org/10.1007/s00402-01903262-z.

37. Xiong Y, Wu Q, Mi B, Zhou W, Liu Y, Liu J, et al. Comparison of efficacy of shock-wave therapy versus corticosteroids in plantar fasciitis: a meta-analysis of randomized controlled trials. Arch Orthop Trauma Surg. 2019;139(4):529-36. https://doi.org/10. 1007/s00402-018-3071-1.

38. Wang YC, Chen SJ, Huang PJ, Huang HT, Cheng YM, Shih C, et al. Efficacy of different energy levels used in focused and radial extracorporeal shockwave therapy in the treatment of plantar fasciitis: a meta-analysis of randomized placebo-controlled trials. J Clin Med. 2019;8. https://doi.org/10.3390/jcm8091497.

39. Huang Q, Yan P, Xiong H, Shuai T, Liu J, Zhu L, et al. Extracorporeal shock wave therapy for treating foot ulcers in adults with type 1 and type 2 diabetes: a systematic review and metaanalysis of randomized controlled trials. Can J Diabetes. 2020;44(2):196-204.e3. https://doi.org/10.1016/j.jcjd.2019.05. 006 .

40. Aldana PC, Khachemoune A. Diabetic foot ulcers: appraising standard of care and reviewing new trends in management. Am J Clin Dermatol. 2020;21(2):255-64. https://doi.org/10.1007/s40257019-00495-x.

41. Samhan AF, Abdelhalim NM. Impacts of low-energy extracorporeal shockwave therapy on pain, pruritus, and health-related quality of life in patients with burn: a randomized placebo-controlled study. Burns. 2019;45(5):1094-101. https://doi.org/10.1016/j.burns.2019. 02.007 .

42. Wang QW, Zhang QY, Gao FQ, Sun W. Focused extra-corporeal shockwave treatment during early stage of osteonecrosis of femoral head. Chin Med J. 2019;132(15):1867-9. https://doi.org/10.1097/ CM9.0000000000000331.

43. Algarni AD, Al Moallem HM. Clinical and radiological outcomes of extracorporeal shock wave therapy in early-stage femoral head osteonecrosis. Adv Orthop. 2018;19:7410246. https://doi.org/10. 1155/2018/7410246.

44. Hao Y, Guo H, Xu Z, Qi H, Wang Y, Lu C, et al. Meta-analysis of the potential role of extracorporeal shockwave therapy in osteonecrosis of the femoral head. J Orthop Surg Res. 2018;3(13(1)):166. https://doi.org/10.1186/s13018-018-0861-7.

45. Xie K, Mao Y, Qu X, Dai K, Jia Q, Zhu Z, et al. High-energy extracorporeal shock wave therapy for nontraumatic osteonecrosis of the femoral head. J Orthop Surg Res. 2018;13(1):25. https://doi. org/10.1186/s13018-017-0705-x.

46. Jun JH, Park GY, Chae CS, Suh DC. The effect of extracorporeal shock wave therapy on pain intensity and neck disability for patients with myofascial pain syndrome in the neck and shoulder: a meta-analysis of randomized controlled trials. Am J Phys Med Rehabil. 2020. https://doi.org/10.1097/PHM.0000000000001493.

47. Yoo JI, Oh MK, Chun SW, Lee SU, Lee CH. The effect of focused extracorporeal shock wave therapy on myofascial pain syndrome of trapezius: a systematic review and meta-analysis. Medicine (Baltimore). 2020;99(7):e19085. https://doi.org/10.1097/MD. 0000000000019085.

48. Zhang L, Cui Y, Liang D, Guan J, Liu Y, Chen X. High-energy focused extracorporeal shock wave therapy for bone marrow edema syndrome of the hip: a retrospective study. Medicine (Baltimore). 2020;99(16):e19747. https://doi.org/10.1097/MD. 0000000000019747.

49. Zhang C, Huang H, Yang L, Duan X. Extracorporeal shock wave therapy for pain relief after arthroscopic treatment of osteochondral lesions of talus. J Foot Ankle Surg. 2020;59(1):190-4. https://doi. org/10.1053/j.jfas.2019.07.015.

50. Schaupper M, Jeltsch M, Rohringer S, Redl H, Holnthoner. Lymphatic vessels in regenerative medicine and tissue engineering. Tissue Eng Part B Rev. 2016;22(5):395-407. https://doi.org/10. 1089/ten.TEB.2016.0034.

51. Sandhofer M, Steinert M, Barsch M. Shock wave therapy in aesthetic medicine. J Ästhet Chir. 2020;13:47-53. https://doi.org/10. 1007/s12631-020-00217-x. 
52. Lohse-Busch H, Marlinghaus E, Reime U, Möwis U. Focused lowenergy extracorporeal shock waves with distally symmetric polyneuropathy (DSPNP): a pilot study. NeuroRehabilitation. 2014;1(35(2)):227-33. https://doi.org/10.3233/NRE-141116.

53. Kim JC, Jung SH, Lee SU, Lee SY. Effect of extracorporeal shockwave therapy on carpal tunnel syndrome: a systematic review and meta-analysis of randomized controlled trials. Medicine (Baltimore). 2019;98(33):e16870. https://doi.org/10.1097/MD. 0000000000016870 .

54. Campbell JD, Trock BJ, Oppenheim AR, Anusionwu I, Gor RA, Burnett AL. Meta-analysis of randomized controlled trials that assess the efficacy of low-intensity shockwave therapy for the treatment of erectile dysfunction. Ther Adv Urol. 2019;11: 1756287219838364. https://doi.org/10.1177/1756287219838364 eCollection 2019 Jan-Dec.

55. Sokolakis I, Dimitriadis F, Teo P, Hatzichristodoulou G, Hatzichristou D, Giuliano F. The basic science behind lowintensity extracorporeal shockwave therapy for erectile dysfunction: a systematic scoping review of pre-clinical studies. J Sex Med. 2019;16(2):168-94. https://doi.org/10.1016/j.jsxm.2018.12. 016.

56. Sokolakis I, Hatzichristodoulou G. Clinical studies on low intensity extracorporeal shockwave therapy for erectile dysfunction: a systematic review and meta-analysis of randomised controlled trials. Int J Impot Res. 2019;31(3):177-94. https://doi.org/10.1038/ s41443-019-0117-z.

57. Dong L, Chang D, Zhang X, Li J, Yang F, Tan K, et al. Effect of low-intensity extracorporeal shock wave on the treatment of erectile dysfunction: a systematic review and meta-analysis. Am J Mens Health. 2019;13(2):1557988319846749. https://doi.org/10.1177/ 1557988319846749 .

58. Vinay J, Moreno D, Rajmil O, Ruiz-Castañe E, Sanchez-Curbelo J. Penile low intensity shock wave treatment for PDE5I refractory erectile dysfunction: a randomized double-blind sham-controlled clinical trial. World J Urol. 2020. https://doi.org/10.1007/s00345020-03373-y.

59. Sramkova T, Motil I, Jarkovsky J, Sramkova K. Erectile dysfunction treatment using focused linear low-intensity extracorporeal shockwaves: single-blind, sham-controlled, randomized clinical trial. Urol Int. 2020;104(5-6):417-24. https://doi.org/10.1159/ 000504788.

60. Chung E, Lee J, Liu CC, Taniguchi H, Zhou HL, Park HJ. Clinical practice guideline recommendation on the use of low intensity extracorporeal shock wave therapy and low intensity pulsed ultrasound shock wave therapy to treat erectile dysfunction: the AsiaPacific Society for Sexual Medicine Position Statement. World J Mens Health. 2020; https://wjmh.org/DOIx.php?id=10.5534/ wjmh.200077.

61. Yuan P, Ma D, Zhang Y, Gao X, Liu Z, Li R, et al. Efficacy of lowintensity extracorporeal shock wave therapy for the treatment of chronic prostatitis/chronic pelvic pain syndrome: a systematic review and meta-analysis. Neurourol Urodyn. 2019;38(6):1457-66. https://doi.org/10.1002/nau.24017.

62. Guu SJ, Geng JH, Chao IT, Lin HT, Lee YC, Juan YS, et al. Efficacy of low-intensity extracorporeal shock wave therapy on men with chronic pelvic pain syndrome refractory to 3-As therapy. Am J Mens Health. 2018;12(2):441-52. https://doi.org/10.1177/ 1557988317736585 .

63. Guu SJ, Liu CC, Juan YS, Li CC, Tsai CC. The 12-month followup of the low-intensity extracorporeal shockwave therapy in the treatment of patients with chronic pelvic pain syndrome refractory to 3-As medications. Aging Male. 2019:1-8. https://doi.org/10. 1080/13685538.2019.1597341.

64. Matthews BG, Hurn SE, Harding MP, Henry RA, Ware RS. The effectiveness of non-surgical interventions for common plantar digital compressive neuropathy (Morton's neuroma): a systematic review and meta-analysis. J Foot Ankle Res. 2019;12:12. https://doi. org/10.1186/s13047-019-0320-7.

65. Park HJ, Hong J, Piao Y, Shin HJ, Lee SJ, Rhyu IJ, et al. Extracorporeal shockwave therapy enhances peripheral nerve remyelination and gait function in a crush model. Adv Clin Exp Med. 2020. https://doi.org/10.17219/acem/122177.

66. Sağir D, Bereket C, Onger ME, Bakhit N, Keskin M, Ozkan E. Efficacy of extracorporeal shockwaves therapy on peripheral nerve regeneration. J Craniofac Surg. 2019;30(8):2635-9. https://doi.org/ 10.1097/SCS.000000000000567.

67. Lee CH, Lee SH, Yoo JI, Lee SU. Ultrasonographic evaluation for the effect of extracorporeal shock wave therapy on gastrocnemius muscle spasticity in patients with chronic stroke. PM R. 2019;11(4): 363-71. https://doi.org/10.1016/j.pmrj.2018.08.379.

68. Cabanas-Valdés R, Serra-Llobet P, Rodriguez-Rubio PR, Lópezde-Celis C, Llauró-Fores M, Calvo-Sanz J. The effectiveness of extracorporeal shock wave therapy for improving upper limb spasticity and functionality in stroke patients: a systematic review and meta-analysis. Clin Rehabil. 2020;34(9):1141-56. https://doi.org/ 10.1177/0269215520932196.

69. Hsieh CK, Chang CJ, Liu ZW, Tai TW. Extracorporeal shockwave therapy for the treatment of knee osteoarthritis: a meta-analysis. Int Orthop. 2020;44(5):877-84. https://doi.org/10.1007/s00264-02004489-x.

70. Ma H, Zhang W, Shi J, Zhou D, Wang J. The efficacy and safety of extracorporeal shockwave therapy in knee osteoarthritis: a systematic review and meta-analysis. Int J Surg. 2020;75:24-34. https:// doi.org/10.1016/j.ijsu.2020.01.017.

71. Cenik F, Mähr B, Palma S, Keilani M, Nowotny T, Crevenna R. Role of physical medicine for cancer rehabilitation and return to work under the premise of the "Wiedereingliede rungsteilzeitgesetz”. Wien Klin Wochenschr. 2019;131(19-20): 455-61. https://doi.org/10.1007/s00508-019-1504-7.

72. Crevenna R, Kainberger F, Wiltschke C, Marosi C, Wolzt M, Cenik F, et al. Cancer rehabilitation: current trends and practices within an Austrian University Hospital Center. Disabil Rehabil. 2020;42(1): 2-7. https://doi.org/10.1080/09638288.2018.1514665.

73. Pleiner J, Crevenna R, Langenberger H, Keilani M, Nuhr M, Kainberger F, et al. Extracorporeal shockwave treatment is effective in calcific tendonitis of the shoulder. A randomized controlled trial. Wien Klin Wochenschr. 2004;116(15-16):536-41. https:// doi.org/10.1007/BF03217707.

74. Saito S, Ishii T, Kamogawa Y, Watanabe R, Shirai T, Fujita Y, et al. Extracorporeal shock wave therapy for digital ulcers of systemic sclerosis: a phase 2 pilot study. Tohoku J Exp Med. 2016;238(1): 39-47. https://doi.org/10.1620/tjem.238.39.

75. Mickel M, Kunstfeld R, Crevenna R. Granuloma annulare and radial pulse therapy: preliminary findings. J Clin Aesthet Dermatol. 2018;11(1):32-4.

76. Wagner B, Ay C, Mickel M, Crevenna R. First application of focused low-energy extracorporeal shockwave therapy in a patient with severe hemophilia A and plantar fasciitis. Wien Klin Wochenschr. 2020. https://doi.org/10.1007/s00508-020-01687-w.

Publisher's Note Springer Nature remains neutral with regard to jurisdictional claims in published maps and institutional affiliations. 